\title{
ON BEHAVIOR OF SOLVABLE IDEALS OF LIE ALGEBRAS UNDER OUTER DERIVATIONS
}

\author{
ANATOLIY P. PETRAVCHUK
}

\begin{abstract}
Let $L$ be a finite dimensional Lie algebra over a field $F$. It is well known that the solvable radical $S(L)$ of the algebra $L$ is a characteristic ideal of $L$ if $\operatorname{char} \mathrm{F}=0$ and there are counterexamples to this statement in case char $\mathrm{F}=\mathrm{p}>0$. We prove that the sum $S(L)$ of all solvable ideals of a Lie algebra $L$ (not necessarily finite dimensional) is a characteristic ideal of $L$ in the following cases: 1) char F $=0 ; 2) S(L)$ is solvable and its derived length is less than $\log _{2} p$. Some estimations (in characteristic 0 ) for the derived length of ideals $I+D(I)+\cdots+D^{k}(I)$ are obtained where $I$ is a solvable ideal of $L$ and $D \in \operatorname{Der}(L)$.
\end{abstract}

\section{INTRODUCTION}

Let $L$ be a finite dimensional Lie algebra over a field of characteristic 0 . It is well-known that its solvable radical $S(L)$ is a characteristic ideal of $L$, i.e. $D(S(L)) \subseteq S(L)$ for every derivation $D \in \operatorname{Der}(L)$ (see, for example, [2, Ch.III, Th.6.7). This result breaks down in characteristic $p>0$ (see [2], p.74-75). The noted counter-example has solvable radical of derived length $\left[\log _{2} p\right]+1$ (because it is the tensor product of a simple Lie algebra over a field $F$ of characteristic $p$ and the group algebra $F[G]$ where $G$ is the cyclic group of order $p$ ). We prove that the solvable radical $S(L)$ is a characteristic ideal if its derived length is less that $\log _{2} p$. The same result is true for infinite dimensional Lie algebras if we denote by $S(L)$ the sum of all solvable ideals of $L$. This sum can be considered as an analogue of solvable radical of the algebra $L$ (the ideal $S(L)$ is locally solvable while the sum of all locally solvable ideals of $L$ can be not locally solvable [5]). If $L$ is an (infinite dimensional) Lie algebra over a field of characteristic 0 we prove that $S(L)$ is always an characteristic ideal of the algebra $L$.

Derivations of Lie algebras and associative algebras were studied by many authors (see for example [6], [3], [1]). In particular, the behavior of locally nilpotent ideals of Lie algebras (in characteristic 0) under derivations was studied in [3], where a passage from a derivation to an automorphism of a Lie algebra was used.

The main results of this paper can be easily deduced from Theorem 11. where behavior of solvable ideals of Lie algebras (not necessarily finite dimensional) is studied under outer derivations of $L$. Let $I$ be a solvable ideal of derived length $n$ of a Lie algebra $L$ and let $D$ be a derivation $D \in \operatorname{Der}(L)$. It is proved that the ideal $I+D(I)$ is solvable of derived length $\leqslant 2 n$ if char $\mathrm{F}=0$ or $n<\log _{2} p$ where $p=\operatorname{char} \mathrm{F}$

Supported by DFFD, Grant F25.1/095 . 
Ideals of the form $I_{k}=I+D(I)+\cdots+D^{k}(I)$ were also studied where $I$ is a solvable ideal of derived length $n$ of the algebra $L$ and $D \in \operatorname{Der}(L)$. The previous results give an estimation for the derived length of $I_{k}$ of the form $s\left(I_{k}\right) \leqslant 2^{k-1} n$. But the Theorem 3 shows that the growth of the derived length is in fact polynomial, namely $s\left(I_{k}\right) \leqslant f(k)$ for some polynomial $f(x)$ of degree $n$.

The notations in the paper are standard. If $L$ is a Lie algebra over a field $F$ then by $S(L)$ we shall denote the sum of all solvable ideals of $L$. We denote by $s(L)$ the derived length of a solvable Lie algebra $L$. The $k$ th member of the derived series of a Lie algebra $L$ is denoted by $L^{(k)}$, in particular, $L^{(0)}=L, L^{(1)}=[L, L], L^{(n)}=\left[L^{(n-1)}, L^{(n-1)}\right]$. In this notations $s(L)=0$ if and only if $L=0$. If $X$ is an $F$-subspace of a Lie algebra $L$ then $X^{1}=X, X^{2}=[X, X], X^{n}=\left[X^{n-1}, X\right]$ are subspaces of $L$. If $D$ is a derivation of a Lie algebra $L$ and $A$ is a $F$-subspace of $L$ then we denote for convenience $D^{0}(A)=A, D^{k}(A)=D\left(D^{k-1}(A)\right)$ for $k \geqslant 1$.

\section{ON CHARACTERISTICITY OF THE SOlVABLE RADiCAL OF A LiE} ALGEBRA

In the next Lemma we collect for convenience some known facts which will be often used in the sequel.

Lemma 1. Let $L$ be a Lie algebra over an arbitrary field $F$, let $D$ be its derivation. Then

1) for any ideal $I$ of the algebra $L$ the $F$-subspace $I+D(I)+\cdots+D^{n}(I)$ is an ideal of $L$ for every $n=1,2, \ldots$;

2) for any elements $x, y \in L$ it holds the Leibnitz's rule:

$$
D^{k}([x, y])=\sum_{s=0}^{k}\left(\begin{array}{l}
k \\
s
\end{array}\right)\left[D^{s}(x), D^{k-s}(y)\right] .
$$

Lemma 2. Let $L$ be a Lie algebra over an arbitrary field, let $I$ be an ideal of the algebra $L$. Then for any derivation $D \in \operatorname{Der}(L)$ it holds $D^{m}\left(I^{(s)}\right) \subseteq I$ for every $m \leqslant 2^{s}-1$.

Proof. Induction on $s$. If $s=1$, then

$$
D\left(I^{(1)}\right)=D([I, I]) \subseteq[D(I), I]+[I, D(I)] \subseteq I
$$

because $I$ is an ideal of $L$. Let the statement of Lemma be true for $s-1$, let us prove it for $s$. Take an arbitrary positive integer $m$ such that $m \leqslant 2^{s}-1$. Then for arbitrary elements $x, y \in I^{(s-1)}$ it holds

$$
D^{m}([x, y])=\sum_{i=0}^{m}\left(\begin{array}{c}
m \\
i
\end{array}\right)\left[D^{i}(x), D^{m-i}(y)\right] .
$$

Since $i+(m-i)=m \leqslant 2^{s}-1$, then at least one of the numbers $i$ or $m-i$ does not exceed $2^{s-1}-1$. As elements $x, y$ lie in $I^{(s-1)}$, we have by the induction hypothesis that either $D^{i}(x)$ or $D^{m-i}(y)$ belong to the ideal $I$. But then from (1) it follows that $D^{m}([x, y]) \in I$. Thus $D^{m}\left(I^{(s)}\right) \subseteq I$ for every nonnegative integers $m \leqslant 2^{s}-1$. The Lemma is proved. 
Lemma 3. Let $L$ be a Lie algebra over a field $F$ and $I$ be an ideal of $L$. If char $F$ does not divide the binomial coefficient $\left(2_{2^{k-1}}^{2^{k}}\right)$, then for every $D \in \operatorname{Der}(L)$ it holds

$$
\left[D^{2^{k-1}}\left(I^{(k-1)}\right), D^{2^{k-1}}\left(I^{(k-1)}\right)\right] \subseteq D^{2^{k}}\left(I^{(k)}\right)+I .
$$

Proof. Let $x, y \in I^{(k-1)}$ be arbitrary elements. Then

$$
D^{2^{k}}([x, y])=\sum_{s=0}^{2^{k}}\left(\begin{array}{c}
2^{k} \\
s
\end{array}\right)\left[D^{s}(x), D^{2^{k}-s}(y)\right] .
$$

Rewrite the last sum as a sum of two summands:

$$
\begin{gathered}
D^{2^{k}}([x, y])=\left(\begin{array}{c}
2^{k} \\
2^{k-1}
\end{array}\right)\left[D^{2^{k-1}}(x), D^{2^{k-1}}(y)\right]+ \\
+\sum_{s=0, s \neq 2^{k-1}}^{2^{k}}\left(\begin{array}{c}
2^{k} \\
s
\end{array}\right)\left[D^{s}(x), D^{2^{k}-s}(y)\right]
\end{gathered}
$$

In the second summand of the righthand side of this formula either the number $s$ or the number $2^{k}-s$ does not exceed $2^{k-1}-1$ and therefore by Lemma 2 the sum $\sum_{s=0, s \neq 2^{k-1}}^{2^{k}}\left(\begin{array}{c}2^{k} \\ s\end{array}\right)\left[D^{s}(x), D^{2^{k}-s}(y)\right]$ lies in the ideal $I$. Further, by conditions of Lemma char $\mathrm{F}$ does not divide the binomial coefficient $\left(2^{2^{k-1}}\right)$, so from the equality (2), taking into account the arbitrary choice of $x$ and $y$, we obtain

$$
\left[D^{2^{k-1}}\left(I^{(k-1)}\right), D^{2^{k-1}}\left(I^{(k-1)}\right)\right] \subseteq D^{2^{k}}\left(I^{(k)}\right)+I .
$$

Lemma 4. An odd prime number $p$ does not divide the binomial coefficients $\left(\begin{array}{l}2 \\ 1\end{array}\right),\left(\begin{array}{c}2^{2} \\ 2\end{array}\right), \ldots,\left(\begin{array}{c}2^{n} \\ 2^{n-1}\end{array}\right)$ if and only if $n<\log _{2} p$.

Proof. Let an odd prime number $p$ do not divide the above mentioned binomial coefficients and $n \geqslant \log _{2} p$. Then we have $p<2^{n}$ and therefore the number $p$ lies in some interval $\left(2^{k-1}, 2^{k}\right)$ for some $k<n$. It is easy to see that the prime number $p$ does divide the binomial coefficient $\left({ }_{2^{k-1}}^{2^{k}}\right)=\frac{1 \cdot 2 \cdots 2^{k}}{\left(1 \cdot 2 \cdots \cdot 2^{k-1}\right)^{2}}$ because $p$ divides the numerator and does not divide the denominator of this fraction. This contradicts to our assumption and therefore $n<\log _{2} p$. Let now $n<\log _{2} p$. Then $p>2^{n}$ and the number $p$ does not divide all the binomial coefficients $\left(\begin{array}{l}2 \\ 1\end{array}\right),\left(\begin{array}{c}2^{2} \\ 2\end{array}\right), \ldots,\left(\begin{array}{c}2^{n} \\ 2^{n-1}\end{array}\right)$. The proof is complete.

Theorem 1. Let $L$ be a Lie algebra over a field $F$ and let $I$ be its solvable ideal of derived length $n$. Then the ideal $I+D(I)$ is solvable and its derived length $\leqslant 2 n$ in the following cases: 1) char $\mathrm{F}=0$; 2) $n<\log _{2} p$ where $p=\operatorname{char} \mathrm{F}>0$.

Proof. If $p=2$ then the statement is true because in this case $s(I)=n=0$ i.e. $I=0$. So, we assume that char $\mathrm{F}=0$ or $\operatorname{char} \mathrm{F}=\mathrm{p}>2$. Denote $J=I+D(I)$ and show that for every positive integer $k,(k \leqslant n)$ it holds 
$J^{(k)} \subseteq D^{2^{k}}\left(I^{(k)}\right)+I$. We shall prove this inclusion by induction on $k$. If $k=1$ then

$$
J^{(1)}=[J, J]=[I+D(I), I+D(I)] \subseteq I+[D(I), D(I)] .
$$

For arbitrarily chosen elements $x, y \in I$ we have by the Leibnitz's rule

$$
[D(x), D(y)]=\frac{1}{2}\left\{D^{2}([x, y])-\left[D^{2}(x), y\right]-\left[x, D^{2}(y)\right]\right\} .
$$

Thus $[D(I), D(I)] \subseteq D^{2}\left(I^{(1)}\right)+I$ and therefore $J^{(1)} \subseteq D^{2}\left(I^{(1)}\right)+I$. Assuming that the inclusion is proved for $k-1$ we shall prove it for $k$. By inductive hypothesis $J^{(k-1)} \subseteq D^{2^{k-1}}\left(I^{(k-1)}\right)+I$. Therefore

$$
J^{(k)}=\left[J^{(k-1)}, J^{(k-1)}\right] \subseteq\left[D^{2^{k-1}}\left(I^{(k-1)}\right)+I, D^{2^{k-1}}\left(I^{(k-1)}\right)+I\right] .
$$

Since $I$ is an ideal of $L$, it follows from above the inclusion

$$
J^{(k)} \subseteq\left[D^{2^{k-1}}\left(I^{(k-1)}\right), D^{2^{k-1}}\left(I^{(k-1)}\right)\right]+I .
$$

From the conditions of Theorem and by Lemma 4 it follows that char F does not divide the binomial coefficient $\left(2_{2^{k-1}}^{2^{k}}\right)$. Then by Lemma 3 we obtain

$$
\left[D^{2^{k-1}}\left(I^{(k-1)}\right), D^{2^{k-1}}\left(I^{(k-1)}\right)\right] \subseteq D^{2^{k}}\left(I^{(k)}\right)+I
$$

and therefore $J^{(k)} \subseteq D^{2^{k}}\left(I^{(k)}\right)+I$. Put now in the last inclusion $k=n=$ $s(I)$ where $s(I)$ is the derived length of $I$. Then it holds

$$
J^{(n)} \subseteq D^{2^{n}}\left(I^{(n)}\right)+I=D^{2^{n}}(0)+I=I .
$$

But then $J^{(2 n)} \subseteq I^{(n)}=0$, i.e. $J=I+D(I)$ is a solvable ideal of $L$ of derived length $\leqslant 2 n$. The proof is complete.

The next Theorem is the main result of the paper. It follows immediately from Theorem 1.

Theorem 2. Let $L$ be a Lie algebra over a field $F$ and let $S(L)$ be the sum of all solvable ideals of $L$. Then $S(L)$ is a characteristic ideal of the algebra $L$ in the following cases: 1) char $\mathrm{F}=0$; 2) $S(L)$ is solvable of derived length $<\log _{2} p$ where $p=$ char $\mathrm{F}>0$.

\section{ON DERIVED LENGTH OF IDEALS $I+D(I)+\cdots D^{k}(I)$}

Lemma 5. Let $L$ be a Lie algebra over a field $F$ of characteristic 0 and $I$ be an abelian ideal of $L$. Then for every derivation $D \in \operatorname{Der}(L)$ the ideal $J_{k}=I+D(I)+\cdots+D^{k}(I)$ is solvable of derived length $\leqslant k+1$ for any positive integer $k$.

Proof. Let us show by induction on $k$ that $\left[D^{k}(I), D^{k}(I)\right] \subseteq J_{k-1}$, where $J_{k-1}=I+D(I)+\cdots+D^{k-1}(I)$. If $k=1$, then $[D(I), D(I)] \subseteq D^{2}([I, I])+$ $[D(I), I]+[I, D(I)] \subseteq I$ because $I$ is an ideal of $L$. In our notations $J_{0}=$ $I+D^{0}(I)=I$ and the base of induction is proved. Assume that it holds the inclusion $\left[D^{k-1}(I), D^{k-1}(I)\right] \subseteq J_{k-2}$. Take arbitrary elements $x, y \in I$ and consider the equality

$$
0=D^{2 k}([x, y])=\sum_{s=0}^{2 k}\left(\begin{array}{c}
2 k \\
s
\end{array}\right)\left[D^{s}(x), D^{2 k-s}(y)\right] .
$$


After carrying the central summand from the right side to the left side we obtain

$$
-\left(\begin{array}{c}
2 k \\
k
\end{array}\right)\left[D^{k}(x), D^{k}(y)\right]=\sum_{s=0, s \neq k}^{2 k}\left(\begin{array}{c}
2 k \\
s
\end{array}\right)\left[D^{s}(x), D^{2 k-s}(y)\right] .
$$

Since either $s<k$ or $2 k-s<k$ in the later sum, it is obvious that all summands in the right side of this equality lie in $J_{k-1}$ (by the induction hypothesis). But then $\left[D^{k}(x), D^{k}(y)\right] \in J_{k-1}$, which implies the inclusion $\left[D^{k}(I), D^{k}(I)\right] \in J_{k-1}$ because elements $x, y$ were arbitrarily chosen. As $J_{k}=J_{k-1}+D^{k}(I)$ we obtain the inclusion $\left[J_{k}, J_{k}\right] \subseteq J_{k-1}$, which means that the ideal $J_{k}$ is solvable of derived length $\leqslant k+1$.

The next statement can be easily obtained from elementary properties of linear recurrence relations (see, for example [4], §3.3.3)

Lemma 6. Let $a_{n}-a_{n-1}=f(n), a_{0}=c, n \geqslant 1$ be a real nonhomogeneous first-order recurrence relation where $f(x)$ is a polynomial of degree $k$ and $a_{0}=c$ is an initial condition. Then this relation has a unique solution which is of the form $\left\{a_{n}=\phi(n)\right\}$ where $\phi(x)$ is a polynomial of degree $k+1$.

Lemma 7. Let $a_{0}, a_{1}, a_{2}, \ldots, a_{n}, \ldots$ be a given sequence of real numbers that satisfy inequalities $a_{n} \leqslant a_{n-1}+f(n), n=1,2, \ldots$, where $f(x)$ is a given polynomial of degree $k$ Then there exists a polynomial $g(x)$ of degree $k+1$ such that $a_{n} \leqslant g(n)$ for all $n=0,1,2, \ldots$

Proof. Consider the sequence $b_{0}, b_{1}, b_{2}, \ldots, b_{n}, \ldots$, where $b_{0}=a_{0}, b_{1}=a_{0}+$ $f(1), b_{n}=b_{n-1}+f(n)$ for all $n$. For this sequence, which obviously satisfies the recurrence relation $b_{n}=b_{n-1}+f(n)$ with the initial condition $b_{0}=a_{0}$, one can find by Lemma 6 the unique solution of the form $b_{n}=g(n)$ where $g(x)$ is a polynomial of degree $k+1$. Further, note that $a_{0} \leqslant b_{0}$ and by conditions of Lemma $a_{1} \leqslant a_{0}+f(1)=b_{1}$. If the inequality $a_{k-1} \leqslant b_{k-1}$ is proved then we have $a_{k} \leqslant a_{k-1}+f(k) \leqslant b_{k-1}+f(k)=b_{k}$. Therefore $a_{n} \leqslant b_{n}=g(n)$ for all $n$. The proof is complete.

Theorem 3. Let $L$ be a Lie algebra over a field of characteristic 0 and let I be its solvable ideal of derived length $n$. Then there exists a polynomial $f_{n}(x)$ of degree $n$ such that for every derivation $D \in \operatorname{Der}(L)$ the ideal $I+D(I)+$ $\cdots+D^{k}(I)$ is solvable of derived length $\leqslant f_{n}(k)$.

Proof. Induction on $n$. If $n=1$ then by Lemma 5 the derived length of the ideal $I_{k}=I+D(I)+\cdots+D^{k}(I)$ does not exceed $k+1$, so one can take $f_{1}(x)=x+1$. Assume that the inequality $s\left(I_{k}\right) \leqslant f_{n-1}(k)$ is proved for some polynomial $f_{n-1}(x)$ of degree $n-1$ provided that $s(I)=n-1$. Denote for convenience by $s_{k}^{(n)}$ the derived length of the ideal $I_{k}$, where $I$ is a solvable ideal of derived length $n$. Take any elements $x, y \in I$ and consider the relation

$$
\begin{gathered}
{\left[D^{k}(x), D^{k}(y)\right]=\left(\begin{array}{c}
2 k \\
k
\end{array}\right)^{-1} \cdot\left\{D^{2 k}([x, y])-\right.} \\
\left.-\sum_{s=0, s \neq k}^{2 k}\left(\begin{array}{c}
2 k \\
s
\end{array}\right)\left[D^{s}(x), D^{2 k-s}(y)\right]\right\}
\end{gathered}
$$


which can be easily deduced from (1) where $m=2 k$. Since at least one of numbers $s$ or $2 k-s$ is less than $k$, we obtain that the sum $\sum_{s=0, s \neq k}^{2 k}\left(\begin{array}{c}2 k \\ s\end{array}\right)\left[D^{s}(x), D^{2 k-s}(y)\right]$ belongs to $I_{k-1}$. The sum $D^{2 k}\left(I^{(1)}\right)+I_{k-1}$ is contained in the ideal

$$
I^{(1)}+D\left(I^{(1)}\right)+\ldots+D^{2 k}\left(I^{(1)}\right)+I_{k-1}
$$

whose derived length does not exceed $s_{2 k}^{(n-1)}+s_{k-1}^{(n)}$. So, from the relation (3) we obtain in our notations $s_{k}^{(n)}-1 \leqslant s_{k-1}^{(n)}+s_{2 k}^{(n-1)}$. By induction hypothesis it holds $s_{2 k}^{(n-1)} \leqslant f_{n-1}(2 k)$ and therefore $s_{k}^{(n)} \leqslant s_{k-1}^{(n)}+f_{n-1}(2 k)+1$. Denote $h_{n-1}(x)=f_{n-1}(2 x)+1$. Then $h_{n-1}(x)$ is a polynomial of degree $n-1$. We obtain a recurrence relation $s_{k}^{(n)} \leqslant s_{k-1}^{(n)}+h_{n-1}(k)$. By Lemma 7 it holds $s_{k}^{(n)} \leqslant f_{n}(k)$, where $f_{n}(x)$ is a polynomial of degree $n$. The proof is complete.

\section{REFERENCES}

[1] R. Amayo, I. Stewart, Infinite-dimensional Lie algebras, Noordhoff International Publishing Leyden, 1974, 425p.

[2] N. Jacobson, Lie algebras, Interscience tracts, no.10 (New York, 1962).

[3] B. Hartley, Locally nilpotent ideals of a Lie algebra, Proc. Cambridge Phil. Soc., 63 (1967), 257-272.

[4] Kenneth H. Rosen (Editor-in-Chief), Handbook of Discrete and Combinatorial Mathematics, CRC Press, (2000).

[5] V.N.Latyshev, A.V.Mikhalev, S.A.Pikhtilkov, On the sum of locally solvable ideals of Lie algebras, (English. Russian original) Mosc. Univ. Math. Bull. 58, No. 3, 18-20 (2003); translation from Vestn. Mosk. Univ. Ser. I, No. 3, 29-32 (2003).

[6] George B. Seligman, Characteristic ideals and the structure of Lie algebras, Proceedings of the AMS, vol. 8, no.1, (1957), 159-164.

Department of Algebra and Mathematical Logic, Faculty of Mechanics and Mathematics, Kyiv Taras Shevchenko University, Volodymyrska street, 01033 KYIV, UKRAINE

E-mail address: aptr@univ.kiev.ua 